\title{
Review on Adeno Virus; As a Vaccine Vehicle
}

\author{
Biniam Tadesse $^{1 *}$, Biruk Tesfaye ${ }^{2}$, Ashenafi Kiros ${ }^{1}$ \\ ${ }^{I}$ National Animal Health Diagnostic and Investigation Center, Sebeta, Ethiopia \\ ${ }^{2}$ Addis Ababa University, College of Veterinary Medicine and Agriculture, Bishoftu, Ethiopia
}

*Corresponding Author: Biniam Tadesse, National Animal Health Diagnostic and Investigation Center, Sebeta, Ethiopia, Email: binimanu10@gmail.com

\begin{abstract}
Viral vectors are generally genetically-engineered viruses carrying modified viral DNA or RNA that has been rendered non infectious, but still contain viral promoters and also the transgene. Adenoviruses have shown tremendous promise as delivery vehicles for recombinant vaccination and gene therapy since the adenoviral genome is well characterized, comparatively easy to manipulate and minimum risk of integration into the host genome. Particularly human adenovirus type 5 has been used in the development of veterinary vaccines. Most vectors currently used as vaccine carriers are deleted in E1 gene to make them replication deficient and to reduce vectors' ability to induce death of the infected cells. E1-deleted Adenoviral vectors are generated from so-called molecular cloning of a transgene in a bacterial plasmid. E3 deleted Adenoviral vectors are replication competent and thus less predictable than replication defective vectors. Administration of viral vectors leads to initiation of both innate and adaptive immunity to elicit powerful $B$ cell and $T$ cell responses. For effective veterinary use, extensive research on adenoviral vaccine vectors should be undertaken.
\end{abstract}

Keywords: Adenovirus, Deletion, Recombinant, Tropism, Vaccine, Replication

\section{INTRODUCTION}

Viruses, however, are not cells, are not microorganisms. They possess no functional organelles and are completely dependent on their host for the machinery of energy production and synthesis of macromolecules. Viruses are obligate intracellular parasites which are capable of utilizing the cellular machinery for production of virally encoded proteins. Microbial Vectors called (expression constructs) are specifically for the expression of the transgene in the target cell, and generally have a promoter sequence that drives expression of the transgene. Viruses such as Adenoviruses, Herpes viruses and Pox viruses have been used in a wide range of applications.

Viral vectors are generally geneticallyengineered viruses carrying modified viral DNA or RNA that has been rendered non infectious, but still contain viral promoters and also the transgene. Viral vectors are often designed for permanent incorporation of the insert into the host genome. Thus, viral vectors are biological delivery vehicles which are ideal tool for gene transfer and are often used by molecular biologists to deliver therapeutic genes
(Hilleman, 2002). Adenoviruses are showing substantial promise as vehicles for antigen delivery in the development many veterinary vaccines. Adenoviruses are showing substantial promise as vehicles for antigen delivery for a number of vaccines currently being developed (Rodriguez and Grubman, 2009).

The criteria of selection for viral vectors are the capacity to infect an extensive range of cell types, known to cause mild or no disease and immunological properties of the live vector to elicit an immune response. But viruses that are pathogenic but attenuated by deletion of virulence genes can be used (Gerdts et al., 2006; MacLachlan et al., 2007; Swayne, 2009).

The major advantages of live vectors are that they produce the antigen in its native conformation, which is important for generating neutralizing antibodies and can facilitate delivery of the internalized peptides (antigen) to the ER lumen where they can bind class I MHC proteins and be targeted to the cell surface where they can then elicit a cellular (Tc) response (Mills, 2009).

Adeno viruses have shown tremendous promise as delivery vehicles for recombinant vaccination 
and gene therapy. Adenoviruses are ubiquitous pathogens associated with mild or no diseases in immune competent animals such as birds, mammals, reptiles and amphibians. The ability to infect a wide range of actively dividing and non dividing mammalian cells and to induce a high level of transgene expression made Adenovirus to develop high levels of antigen specific humoral and cell mediated immune responses. The adenoviral genome is well characterized, comparatively easy to manipulate and minimum risk of integration into the host genome. They can be applied systemically as well as through mucosal surfaces and their relative thermo stability facilitates their clinical use (Gao et al., 2006).

Therefore, the objectives of this seminar paper are:

$>$ To outline the basic principles of adenovirus vector construction.

$>$ To provide insight on the application and challenges of adenovirus as a vaccine vector.

\section{ADENO VIRUSSTRUCTURE AND REPLICATION}

\subsection{Virion Property}

Adenoviruses were first isolated from cultures of human adenoid tissue (tissue located at the back of the nose, above the tonsils) and were discovered in 1953. Adenoviruses are medium sized $(90-100 \mathrm{~nm})$, non enveloped, icosahedrally symmetric double stranded DNA viruses. The genome of adenovirus is approximately 34 - $43 \mathrm{~kb}$ and to conserve space, it encodes polypeptides from both DNA strands and uses alternative splicing and different polyadenylation sites (Samuel and Michael, 2007).

The adenovirus capsid is composed primarily of 240 homotrimeric hexon capsomers with twelve Penton capsomers located at each of the twelve fivefold axes of symmetry. The capsid of the virus contains three major proteins: Hexon, Fibre and Penton Base proteins. The Hexon protein provides structural support, but differs in size and immunological properties between serotypes. The fiber protein of adenovirus exhibit high homology among serotypes and occurs as a trimer on the surface of the capsid. The fibre proteins are anchored to the capsid by the penton base proteins, which occur as a pentamer. Together with the fiber protein, it is responsible for the attachment of the virus to cell surfaces (Luci, 1997).

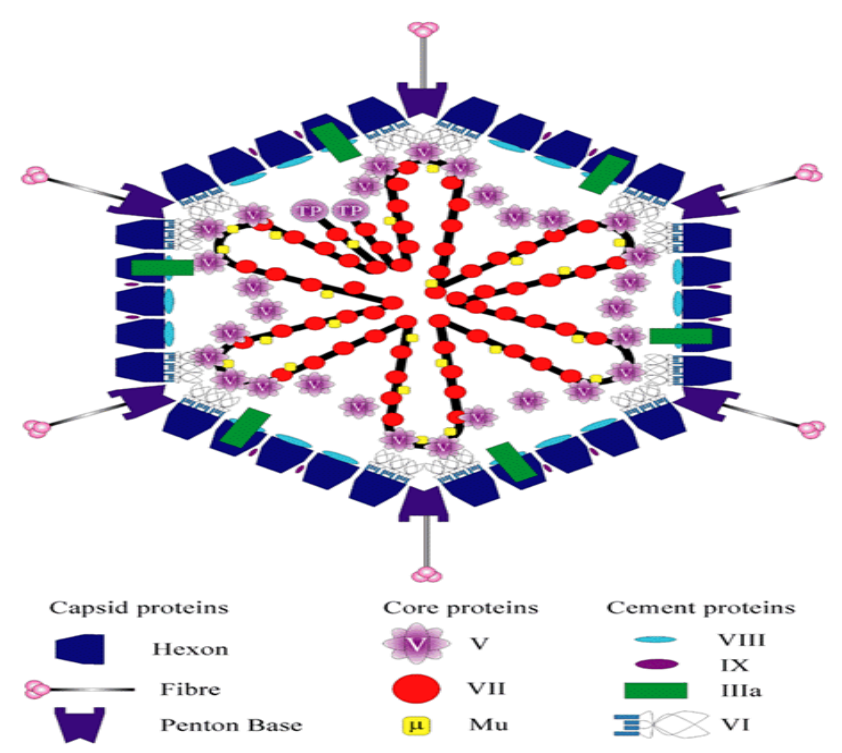

Figure1. Adeno virus structure (Russell, 2001)

Its genome carries five early genes: E1A, E1B, E2, E3, and E4 (Yue, 2000). The E1A gene induces expression of other viral early genes. The E1B gene delays host cell lysis during viral replication and after replication is complete, it induces apoptosis and controls the export of viral transcripts. Deletion of E1 results in viruses that are severely impaired in their ability to replicate and allows for the insertion of approximately $5.1 \mathrm{~kb}$ of new DNA (Danthinne and Imperiale, 2000). 


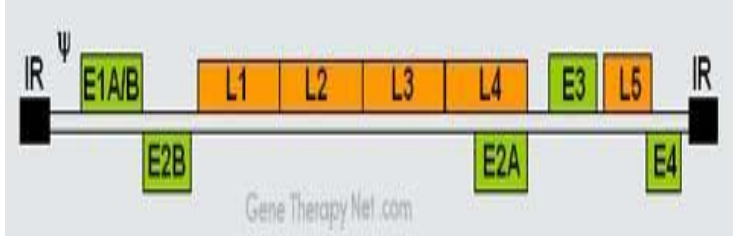

Figure2. Genome organization of adenoviruses (Gene therapy.net.com, 2002)

\subsection{Virus Replication}

Adenoviruses replicate in the nucleus and their replication is facilitated by extensive modulation of the host immune response. Viruses bind to host cell receptors via their penton fibers and enter the cell by endocytosis via clathrin-coated pits (James and Edward, 2011). Clathrinmediated endocytosis followed by partial capsid disassembly upon acidification of the endosome liberates protein VI and promotes membrane disruption (Christopher, 2005; Lynda et al, 2010).

Endosomal escape is modulated by the lytic action of protein VI and transport to the nuclear pore complex involves the microtubuledependent motor after which the nucleocapsid is translocated to the perinuclear envelope along the microtubule network (Magnus, 2002; Wei and Michael, 2003). The outer capsid is then removed and the core comprising the viral genome with its associated histones enters the nucleus where messenger RNA (mRNA) transcription, viral DNA replication, and assembly of virions occur. In the nucleus, the genome is transcribed by cellular RNA polymerase II according to a complex program involving both DNA strands (Flint and Shenk, 1997; James and Edward, 2011).

Replication is divided into two phases: early and late. There are five early (E) transcriptional units (E1A, E1B, E2, E3, and E4). The early genes which are encoded on both strands of the DNA are responsible for expressing mainly non structural regulatory proteins. These proteins are for alteration of expression of the host protein and to avoid premature death of the infected cells by the host immune system. The E1A region of the viral genome encodes proteins that are essential for induction of cell cycle progression (DNA synthesis), protection of infected cells from host antiviral immune defenses and synthesis of viral proteins necessary for viral DNA replication (Meir, 2004).

The late phase starts upon onset of DNA replication by producing structural protein to pack all the genetic material produced by DNA replication (Flint and Shenk, 1997). The late region uses a single promoter for transcription of the five families of late mRNAs (L1 to L5) which are translated into structural proteins, (James and Edward, 2011).

\section{Adeno Virus as a Vaccine Vehicle}

\subsection{Construction of Adenoviral vectors}

Numerous strategies have been developed to construct Adenovirus vectors carrying a foreign gene insert. Traditionally, Adenovirus vectors were constructed using two standard methods. The first method is the in vitro ligation method involving the ligation of a DNA fragment obtained by restriction digesting of a plasmid carrying the foreign gene insert flanked by Adenovirus sequences with a DNA fragment representing the rest of Adenovirus genome (Bett et al., 1994). The second method consisted of homologous recombination in permissive cell lines between two plasmids. The shuttle plasmid which is carrying the foreign gene insert is flanked by Adenovirus sequences for site specific insertion and the genomic plasmid carrying almost the entire Adenovirus genome (Luo et al., 2007).

Alternate approaches have been developed to avoid the limitations of traditional methods. One such strategy is based on the highly efficient homologous recombination machinery of $E$. coli (BJ5183). Adenovirus vectors homologous recombination between a linearized or intact plasmid containing almost an entire Adenovirus genome and a shuttle plasmid containing an exogenous expression cassette flanked by homologous sequences from the site of insertion in the Adenovirus genome generates an infectious clone with a modification and/or insertion in the desired region (Van Olphen and Mittal, 1999).

A similar strategy employing homologous recombination in yeasts has been reported. This strategy involves homologous recombination between Adenovirus DNA and the yeast artificial chromosome vector containing sequences from the left and the right termini of Adenovirus genome, resulting in the generation of a yeast artificial chromosome containing an infectious copy of Adenovirus genome. Transfection of the excised Adenovirus genome into appropriate cells results in generation of infectious virions (Ketner et al., 1994). 


\subsubsection{Deletion}

The adenoviral capsid allows some latitude regarding the length of genome that can be packaged efficiently. Foreign sequences of up to $1.8 \mathrm{~kb}$ can be inserted into the adenoviral genome without necessitating deletions. The ability to identify and selectively delete genes from a pathogen can increase the permitted size of the insert and to modify the biology of adenoviral vectors. To incorporate larger foreign sequences, vectors deleted of E1 and E3 have been developed and they accommodate up to 7.5 $\mathrm{kb}$ of foreign DNA (He, 2000; Saito et al., 1984).

Most vectors currently used as vaccine carriers are deleted in E1 to make them replication deficient and to reduce vectors' ability to induce death of the infected cells. The original E1 deleted Adenoviral vectors were constructed by homologous recombination. Now most E1-

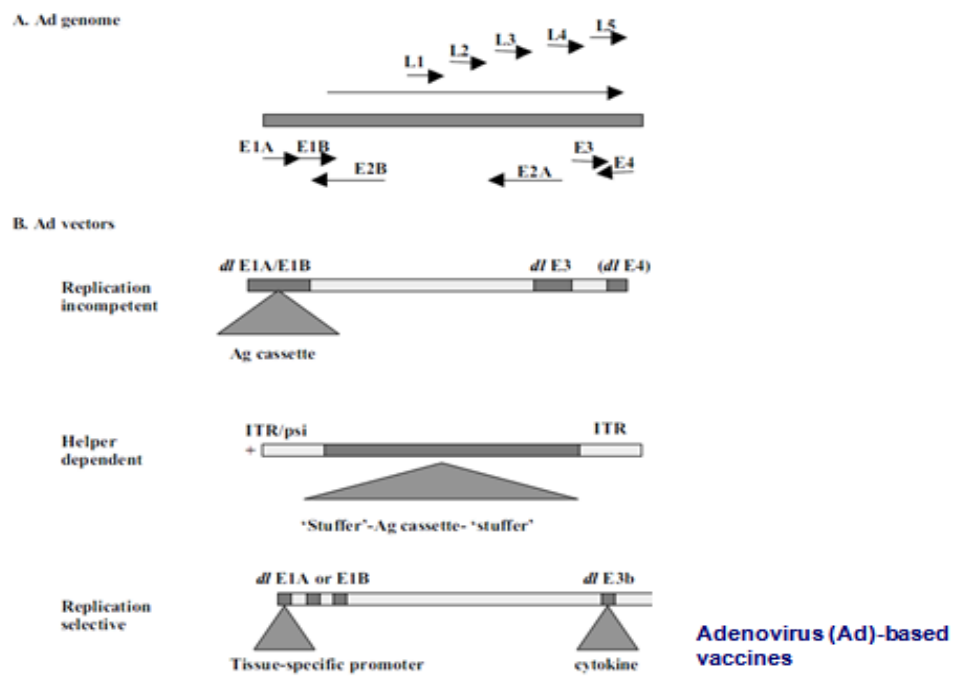

Figure3. Adeno virus based vaccines (Flint and Shenk, 1997)

\subsubsection{Replication Competent Adenoviral Vectors}

A replication competent Adenovirus retains the genetic material in the vector that required for replication in the host cell. The $\mathrm{E} 3$ region can be deleted without affecting the ability to replicate and encode all of the remaining adenoviral antigens in addition to the transgene product, i.e., the vaccine antigen (Zhao, 2003).

The desired immune response from the vaccine antigen can be affected by competition for MHC binding sites or cytokines needed for activation, differentiation and proliferation of $\mathrm{T}$ and $\mathrm{B}$ cells. Furthermore, apoptotic death of the infected cells shortens the duration of antigen presentation by individual infected cells, which in turn could have a negative effect on induction
Adenovirus (Ad)-based vaccines deleted Adenoviral vectors are generated from so-called molecular cloning of a transgene in which the entire Adenoviral genome including the ITRs is carried in a bacterial plasmid, allowing for its propagation in Escherichia coli (He et al., 1998).

E3 deleted Adenoviral vectors achieve higher antigenic loads compared to replication defective vectors and thus result in higher and more sustained transgene product-specific immune responses. E3 deleted Adenoviral vectors are replication competent and thus less predictable than replication defective vectors (Zhao, 2003). The E1-deleted Adenoviral vectors are safer than the E3-deleted Adenoviral vectors since the E3-deleted Adenoviral vectors raises concern about potential toxicity especially if they are used in immune compromised patients. of $\mathrm{CD}^{+} \mathrm{T}$ cells, which are stimulated primarily by peptides derived from denovo synthesized antigens, while potentially favoring activation of $\mathrm{CD} 4^{+} \mathrm{T}$ cells, which are induced by peptides derived from lysosomal cleavage of proteins taken up by phagocytosis or pinocytosis $(\mathrm{He}$, 2000).

\subsubsection{Replication incompetent Adenoviral vectors}

Replication incompetent vectors contain an antigen expression cassette substituted for the deleted E1A-E1B region. These replication incompitant adenoviruses can not replicate because of the deletion of the essential viral E1 gene region containing two genes, E1a and E1b. The removal of the E1 gene region creates room for the vaccine expression cassette and prohibits 
transactivation of viral genes required for viral replication (Lai et al., 2002). In addition to the E1 deletion, E3 region is also deleted to accommodate larger insertions. The viral E3 gene is dispensable for production of recombinant virus as well as the E4 gene which reduces proinflammatory responses in vivo. The antigen expression cassette is inserted into the deleted region together with a 'filler' sequence, so that the overall vector length is approximately the $36 \mathrm{kbp}$ size similar to native Ad, to achieve efficient propagation. Replication defective Adenovirus vectors are extremely safe, stable and easily produced in high titers (Lusky, 1998).

There are also replication selective Adenoviruses that contain deletions in E1A or E1B to render their propagation selective in cancer cells deficient in p53 or pRB function. E1A promoter is substituted with tissue specific promoters so that productive virus infection occurs selectively in tumors having appropriate factors for activity of these promoters (Konstantin etal., 2001).

\subsubsection{Adeno associated viral vectors}

Adeno Associated Viruses are non enveloped, single stranded DNA viruses with a diameter of 18-25 nm. They belong to the Parvoviridae family and are classified in the Dependovirus genus. The virus particle is composed of one single molecule of the viral genome of either positive or negative sense packaged in to an icosahedral capsid. Adeno Associated Viruses are very resistant to extreme conditions of $\mathrm{pH}$, detergent and temperature, making them easy to manipulate. Adeno Associated Viruses are replication defective and require co infection with a helper virus, such as adenovirus to undergo a productive infection characterized by viral gene expression, genom replication and virion production. It has been shown that Adeno associated virus replication can be facilitated by selected proteins from the adenovirus genome (Matsushita et al., 1998).

Adeno Associated Virus vectors are simple, versatile and safe and successfully used for the long term expression of therapeutic genes in animal models and patients. Furthermore, studies have demonstrated that recombinant Adeno associated vectors can evade the immune response and mediate a durable expression of transgene in vivo (Fisher et al., 1997). However, in some circumstances, the Adeno associated virus vector may initiate adaptive immune responses to the transgene product (Manning et al., 1997).

\subsubsection{Tropism alteration}

The tropism of Adenoviral vectors can be altered by genetic engineering of the fiber knob. A large portion of the C-terminus of HAd5 fiber is replaced with the $\sigma 1$ protein of Reovirus type 3 results in production a stable vector. Reovirus receptor junctional adhesion molecule 1 uses for viral entry and thus show improved transduction of certain cell types, including dendritic cells results in improved induction of $\mathrm{CD}^{+} \mathrm{T}$ cells in vitro or in vivo (Mercier, S. et al., 2004; Rea, 2001).

\subsection{Immune Responses Generated by Adenoviral Vectors}

Administration of viral vectors lead to initiation of both innate and adaptive immune responses generated against the transgene and the vector itself. Following systemic administration, adenoviral vectors will attach to the Coxsackie Adenovirus Receptor (CAR) on host cells and are internalized then the viral DNA will enter the nucleus of the cell and is eventually transcribed (Trotman et al., 2000). Immune responses can be triggered by internalized viruses via various Toll-like receptors (Uematsu et al., 2007).

They activate the innate immune system presumably by expressing so called pathogen associated molecular patterns (PAMPs). Production of pro inflammatory cytokines and differentiation of immature dendritic cells into professional antigen-presenting cells initiated when the PAMPs bind to pathogen recognition receptors on host cells. Systemic administration of high doses of AdHu5 vectors into mice or monkeys was shown to trigger rapid release of IL-6, IL-12, and TNF- $\alpha$ and accumulation of transduced macrophages and dendritic cells in lymphatic tissues. Significant levels of cytokines including IL-6 and TNF $\alpha$ are usually detected within 24 hours following Adenoviral vector administration. (Verdino et al., 2010; Schnell, 2001; Bessis et al., 2004)

The potential for Adenoviruses to elicit powerful $\mathrm{B}$ cell and $\mathrm{T}$ cell responses in the mammalian host are the main reason for the use of these vectors in vaccine development. The strong immune responses elicited by these vectors have been linked to their ability to infect immature dendritic cells thereby activating them to become mature antigen presenting dendritic cells thus promoting $\mathrm{T}$ cells responses which are mainly $\mathrm{CD}^{+}{ }^{+} \mathrm{T}$ cells (Morelli et al., 2000). In 
experimental animals' Adenoviral vectors structural proteins (Hong et al., 2003). induce $\mathrm{CD}^{+} \mathrm{T}$ cell responses to different

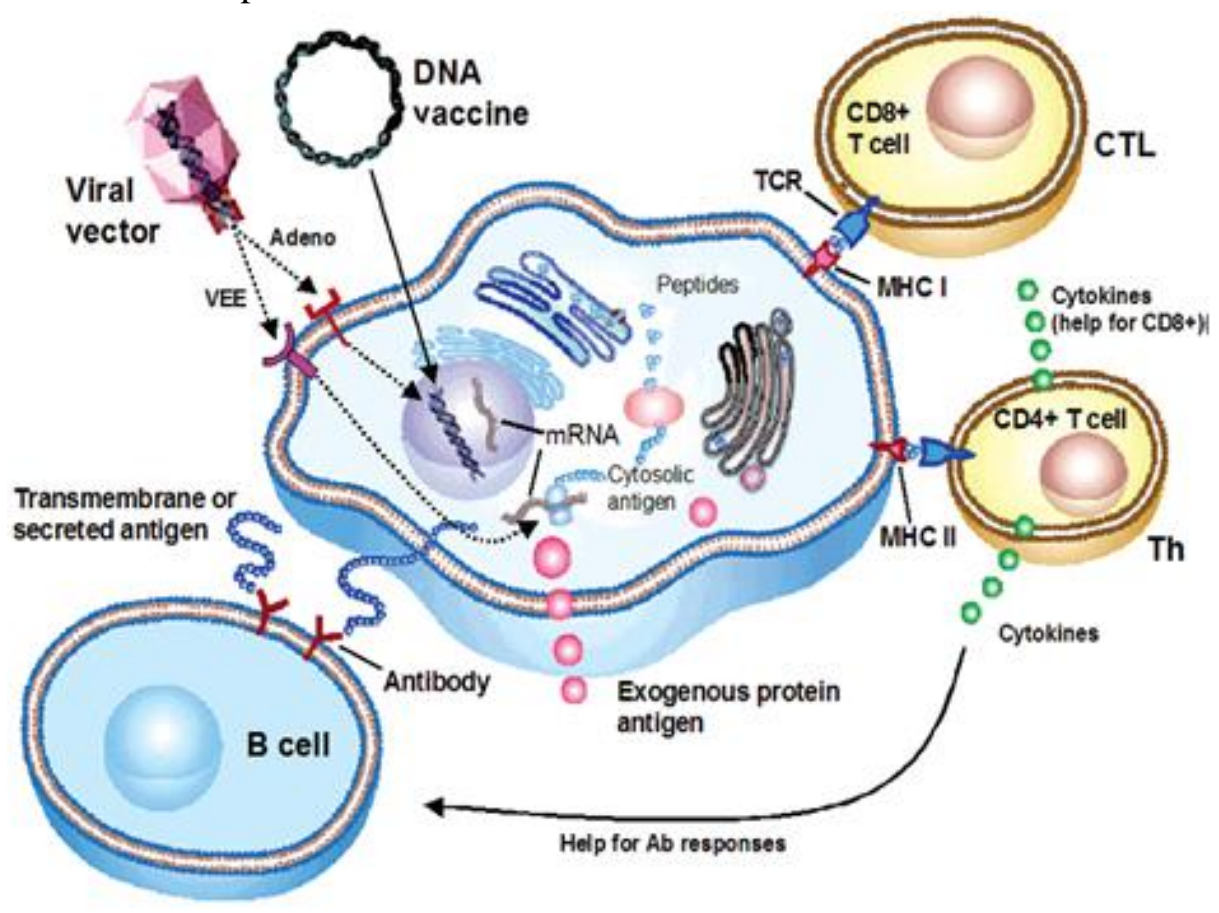

Figure4. Generation of antigen-specific humoral and cellular responses (Andreas et al., 2006)

\subsection{Adenoviral Vectors Currently Used in Veterinary Vaccines}

Adenoviruses have become a vector of choice for delivery and expression of foreign proteins for vaccination particularly human adenovirus type 5. Human serotype adenovirus vaccine Table1.Examples of adenoviruses that have been developed as veterinary vaccine vectors

\begin{tabular}{|l|l|l|l|l|}
\hline Vector & $\begin{array}{l}\text { Animal } \\
\text { Model }\end{array}$ & $\begin{array}{l}\text { Route of } \\
\text { delivery }\end{array}$ & Results of animal studies & Reference \\
\hline $\begin{array}{l}\text { Canine adenovirus serotype 2/ } \\
\text { rabies virus glycoprotein }\end{array}$ & Dog & Subcutaneous & $\begin{array}{l}100 \% \text { protection following } \\
\text { challenge with rabies virus }\end{array}$ & $\begin{array}{l}\text { Hu } \text { et al., } \\
2006\end{array}$ \\
\hline $\begin{array}{l}\text { Human adenovirus serotype } \\
\text { 5/avian influenza virus } \\
\text { haemagglutinin (HA) }\end{array}$ & Chicken & Subcutaneous & $\begin{array}{l}\text { High levels of HA specific } \\
\text { antibodies; High levels of } \\
\text { IFN-gamma }\end{array}$ & $\begin{array}{l}\text { Ramos } \text { et al. } \\
2010\end{array}$ \\
\hline $\begin{array}{l}\text { Replication defective Had 5 } \\
\text { containing capsid polypeptide } \\
\text { from FMD V }\end{array}$ & Pig & Intramuscular & Sufficient protection & $\begin{array}{l}\text { Mayr } \text { et al., } \\
1999\end{array}$ \\
\hline $\begin{array}{l}\text { Fowl adenovirus serotype 1/ } \\
\text { VP2 protein of infectious } \\
\text { bursal disease virus }\end{array}$ & Chicken & Oro nasal & Complete protection & $\begin{array}{l}\text { Francois } \text { et } \\
\text { al., 2004 }\end{array}$ \\
\hline $\begin{array}{l}\text { Replication defective HAd 5 } \\
\text { expressing HA from swine } \\
\text { influenza virus }\end{array}$ & Pig & Subcutaneous & $\begin{array}{l}\text { Induce both cellular and } \\
\text { humoral response }\end{array}$ & $\begin{array}{l}\text { Wesley } \text { et al., } \\
2004\end{array}$ \\
\hline
\end{tabular}

\subsection{The Potential for Commercial Adenoviral} Vector Based Veterinary Vaccines

Conventional vaccines have played an important role in the control and possible eradication of certain diseases e.g. rinderpest. However, new approaches to vaccine development are urgently required for those diseases where efficacious vaccines do not exist (Domenech et al., 2010). vectors are particularly useful for development of veterinary vaccines (Zeshan et al., 2010). Adenoviruses from various species have been developed as vectors and some examples are listed in Table 1.
Despite years of research into the technologically advanced DNA and viral/ bacterial vector technologies, conventional vaccines based upon either killed or attenuated pathogens or recombinant antigens still form a major part of the veterinary vaccine market today. One of the main reasons for this is the relatively long process leading to commercialization of a new vaccine which 
involves establishing vaccine efficacy and safety prior to obtaining registration, which may be complicated if the vaccines were to be commercialized globally (Flore et al., 2004).

Currently however, veterinary vaccines comprise a fraction of the market size of human vaccines; consequently there are much lower levels of investment into veterinary vaccine research and development. These issues can have a negative impact on the utilization of new technologies in veterinary vaccine development. The commercialization of vaccines whether for human or veterinary use usually begins following extensive testing of the vaccine candidate in animal models and field trials. The final stage of this arduous process would be market authorization which for a veterinary vaccine would involve obtaining permission from veterinary authorities to allow the vaccine to be made available to the relevant consumers (Heldens et al., 2010).

Over the years a number of adenoviral vector based veterinary vaccines have been developed but none are currently licensed. One such Adenoviral vector based veterinary vaccine is currently under consideration for commercialization. This is a replication defective human adenovirus serotype 5 containing the capsid and $3 \mathrm{C}$ protease coding regions of foot and mouth disease virus as a vaccine candidate has been developed and gone through field trials and other testing in preparation for commercial production (Shen, 2009).

\section{Challenges in Application OF ADENOVIRAL VACCINE VECTOR}

Despite the many advantages of this technology there are some potential drawbacks to consider, such as the potential toxicity of adenoviral vaccine vectors. For example, viral based vectors elicit an inflammatory cytokine response (by stimulating both innate and adaptive immune responses), thus promoting harmful side effects in the host. In light of this, these viral vaccine vectors must be thoroughly evaluated for potentially harmful immunological reactions on the host immune system, especially in the context of pre-existing immunity to the vaccine vector prior to conducting field trials (Hartman et al., 2008).

Adenoviral vector based systems are clearly underutilized in veterinary vaccine develop ment despite being well characterized and shown to be potent inducers of protective immune responses. If these vectors are to be used widely to construct veterinary vaccines that can be commercialized, more research needs to be undertaken on this platform, particularly studies focusing on the comparison of this platform with other viral vector platforms and the use of adenoviral vaccines in prime boost immunization protocols with non Adenoviral vector based vaccines (Millar et al., 2007).

Other important issues to be considered when developing Adenoviral vector based on vaccines are the choice of transgene and the stability of the vector genome. Ideally extensive research to identify the most immunogenic antigens of a pathogen should therefore be undertaken before vaccine development begins. In order for a vaccine to be protective, the pathogen specific transgene used in the construction of the vaccine should contain highly immunogenic epitopes otherwise the immune responses generated by the vaccine will be suboptimal. Studies conducted to ensure the stability of the viral vector genome must also be conducted soon after the vaccine is constructed and well before large scale production of the vaccine as it has been reported that mutants which do not express the vaccine transgene can randomly arise when producing large quantities of viral vector based vaccines (Wyatt et al., 2009).

Due to the ubiquitous nature of Adenovirus, a large percentage of animal population has variable levels of neutralizing antibodies know as 'preexisting vector immunity' or 'vector immunity' against more than one Adenovirus serotypes. These neutralizing antibodies are directed against the viral capsid components and adversely affect the uptake of Adenoviral vectors by target cells. Adenovirus neutralizing antibodies persist for years posing a challenge when repeated administrations are necessary (Pichla-Gollon et al., 2009).

Preexisting immunity due to natural infections results in sustained virus neutralizing antibody titers, a major handicap for the successful use of common serotypes of Adenovirus, as carriers for gene replacement therapy or vaccine carriers. Neutralizing antibodies even if present at moderate titers, reduce uptake of the Adenoviral vectors by cells, including antigen presenting cells (Fitzgerald, 2003).

\section{CONCLUSION AND RECOMMENDATIONS}

Advances in molecular virology in concert with viral immunology now allow for the genetic engineering of vectors expressing solely those viral antigens that induce immune correlates of protection. Adenoviruses have moved to the 
forefront of vaccinology and are showing substantial promise as vehicles for antigen delivery for a number of vaccines currently being developed. Due to the versatility and variety of Adenovirus serotypes, they will be valuable tools for developing vaccines against new pathogens and against those to which vaccines have yet to be discovered. Most vectors currently used as vaccine carriers are deleted in E1. This renders the vectors replication defective, to increase the permitted size of the insert and also allows for sustained antigen presentation by reducing the vectors' ability to induce death of the infected cells. The potential for Adenoviruses to elicit powerful B cell and $\mathrm{T}$ cell responses in the mammalian host are the main reason for the use of these vectors in vaccine development. Preexisting Adenoviral vector immunity has been shown to decrease the immunogenicity of Adenoviral based vaccines in animal models. Several strategies to overcome this limitation are being explored and have shown promise in pre-clinical studies. One such strategy involves the use of vectors derived from human Adenoviruses specially HAd 5.

Based on the above conclusion the following recommendations are forwarded:

$\checkmark$ Adenoviral vaccine vectors must be thoroughly evaluated for potentially harmful immunological reactions on the host immune system.

$\checkmark$ Extensive research on Adeno viral vaccine vectors should be undertaken for effective utilization and commercialization of veterinary use.

$\checkmark$ The time taken for licensing and commercialization process of vaccine should be minimized.

Application of such new vector based vaccine technology should be promoted in our country.

\section{REFERENCES}

[1] Andreas, B., Karl, L., Britta, W. and Margaret, L. (2006): Vaccine delivery methods using viral vectors. Molecular pharmaceutics, 4:1

[2] Bessis, N., GarciaCozar, F. and Boissier, M. (2004): Immune responses to gene therapy vectors: Influence on vector function and effectors mechanisms. Gene therapy, 11: 510517.

[3] Bett, A., Haddara, W., Prevec, L. and Graham, F. (1994): An efficient and flexible system for construction of adenovirus vectors with insertions or deletions in early regions 1 and 3 . Proclamation of national academy of science, 91: 8802-6.

ARC Journal of Animal and Veterinary Sciences
[4] Christopher, M. (2005): Adenovirus Protein VI mediates membrane disruption following capsid disassembly. Virology journal, 79(4): 1992-1997

[5] Danthinne, X. and Imperiale, M. (2000): Production of first generation Adenovirus vectors. Review: gene therapy, 7:1707-1708.

[6] Domenech, J., Lubroth, J. and Sumption, K. (2010): Immune Protection in Animals: The Examples of Rinderpest and Foot and Mouth Disease. Journal of comparative pathology, 142: 120-124.

[7] Fisher, K., Jooss, K., Alston, J., Yang, Y., Haecker, S., High, K., Pathak, R., Raper, S. and Wilson, J. (1997): Recombinant Adeno associated virus for muscle directed gene therapy. Nature medicine, 3: 306-312.

[8] Fitzgerald, J. (2003): A simian replication defective adenoviral recombinant vaccine to HIV-1 g. Journal of immunology, 170: 1416 1422.

[9] Flint, J and Shenk, T. (1997): Viral transactivating proteins. Annual review of genetics 31: 177-192.

[10] Flore, P. (2004): Commercialization of veterinary viral vaccines. Animal health research reviews, 5(2): 239-242.

[11] Francois, A., Chevalier, C., Delmas, B., Eterradossi, N., Toquin, D., Rivallan,G. and Langlois, P. (2004): Avian Adenovirus CELO recombinants expressing VP2 of Infectious Bursal Disease Virus Induce Protection against Bursal Disease in Chickens. Vaccine, 22(17): 2351-2360.

[12] Gao, W., Soloff, A. and Lu, X. (2006): Protection of mice and poultry from lethal H5N1 avian influenza virus through Adenovirus based immunization. Journal of virology, 80: 1959-64.

[13] Gene therapy.net.com (2002): Assessment of Adenoviral vector safety and toxicity. Human gene therapy.

[14] Gerdts, V., Mutwiri, G., Tikoo, S. and Babiuk, L. (2006): Mucosal delivery of vaccines in domestic animals. Veterinary research, 37: 487-510.

[15] Hartman, Z., Appledorn, D. and Amalfitano, A. (2008): Adenovirus vector induced innate immune responses: Impact upon efficacy and toxicity in gene therapy and vaccine applications. Virus Research, 132(1-2): 1-14.

[16] He, T., Zhou, S., DaCosta, L., Yu, J., Kinzler, K. and Volgelstein, B. (1998): A simplified system for generating recombinant Adenovirus. Proclamation national academy of science, 95 : 2509-2514.

[17] He, Z. (2000): Viral recombinant vaccines to the E6 and E7 antigens of HPV-16. Virology, 270: $146-161$. 
[18] Heldens, J., Patel, J., Chanter, N., TenThij, G., Gravendijck, M., Schijns, V., Langen, A. and Schetters, T. (2008): Veterinary vaccine development from an industrial Perspective. The veterinary journal, 178(1): 7-20.

[19] Hilleman, M. (2002): Overview of the needs and realities for developing new and improved vaccines in the 21 st century. Inter virology, 45 (4-6): 199-211.

[20] Hong, S., Habib, N., Franqueville, L., Jensen, S. and Boulanger, P. (2003): Identification of Adenovirus penton base neutralizing epitopes by use of sera from patients who had received conditionally replicative Adenovirus for treatment of liver tumors. Virology Journal, 77: 10366.

[21] Hu, R., Zhang, S., Fooks, A., Yuan, H., Liu, Y., Li, H., Tu, C., Xia, X. and Xiao, Y. (2006): Prevention of Rabies virus infection in dogs by a recombinant canine Adenovirus Type-2 Encoding the Rabies virus glycoprotein. Microbes and infection, 8(4): 1090-1097.

[22] James, M. and Edward, D. (2011): Fenner's Veterinary Virology. $4^{\text {th }}$ edition

[23] Ketner, G., Spencer, F. and Tugendreich, S. (1994): Efficient manipulation of the human Adenovirus genome as an infectious yeast artificial chromosome clone. Proclamation of national academy of science, 91: 6186-90.

[24] Konstantin, D., Mohan, K., Karoly, T., Anne, T., Peter, K., Valeri, K. and Williams, S. (2001): Tissue specific, tumor selective replication competent Adenovirus for cancer gene therapy. Journal of virology, 75(7): 3314.

[25] Lai, C., Lai, Y. and Rakoczy, P. (2002): Adenovirus and Adeno- associated virus vectors. DNA Cell Biol., 21: 895-913

[26] Luci, K. (1997): Structural and functional determinants in Adenovirus Type 2 Penton Base recombinant Proteins. Virology journal, 71(11): 8678, 8678

[27] Luo, J., Deng, Z. and Luo, X. (2007): A protocol for rapid generation of recombinant Adenoviruses. National Protocol, 2: 1236-47.

[28] Lusky, M. (1998): In Vitro and In Vivo biology of recombinant Adenovirus vectors with E1, E1/E2A, or E1/E4 deleted. Journal of virology, 72: 2022-2023.

[29] Lynda, C., Raul, A., Alan. P., Angela, B., Iain, M., Stuart, N., and Andrew, B.(2010): TropismModification Strategies for Targeted Gene Delivery Using Adenoviral Vectors. Viruses, 2: 2290-2355.

[30] Maclachlan, N., Balasuriya, U., Davis, N., Collier, M., Johnston, R., Ferraro, G. and Guthrie, A. (2007): Experiences with new generation vaccines against Equine viral arteritis, West Nile disease and African horse sickness. Vaccine, 25 (30): 5577-5582.
[31] Magnus, M. (2002): Unscheduled Expression of Capsid Protein IIIa results in defects in Adenovirus major late mRNA and Protein expression. Virus research, 83: 197

[32] Manning, W., Paliard, X., Zhou, S., Pat Bland, M., Lee, A., Hong, K., Walker, C., Escobedo, J .and Dwarki, V. (1997) Genetic immunization with Adeno associated virus vectors expressing herpes simplex virus type 2 glycoproteins B and D. Journal of virology, 71: 7960-7962.

[33] Matsushita, T., Elliger, S. and Elliger, C. (1998): Adeno associated virus vectors can be efficiently produced without helper virus. Gene therapy, 5: 938-945.

[34] Mayr, G., Chinsangaram, J. and Grubman, M. (1999): Development of replication-defective adenovirus serotype 5 containing the capsid and 3C protease coding regions of foot-and-mouth disease virus as a vaccine candidate. Virology, 263: 496-506.

[35] Meir, O. (2004): Adeno virus endocytosis. Journal of gene med., 6; s152-s1636

[36] Mercier, G. (2004): A chimeric adenovirus vector encoding reovirus attachment protein sigma1 targets cells expressing junctional adhesion molecule 1. Proclamation of national academy of science, 101: 6188-6693.

[37] Mercier, S. (2004): Adenovirus fibre exchange alters cell tropism in vitro but not transgenespecific $\mathrm{T}$ CD8 ${ }^{+}$immune responses in vivo. Journal of virology, 85: 1227-1236.

[38] Millar, J., Dissanayake, D., Yang, T., Grinshtein, N., Evelegh, C., Wan, Y. and Bramson, J. (2007): The magnitude of the CD8+ $\mathrm{T}$ Cell response Produced by recombinant virus vectors is a function of both the antigen and the vector. Cellular immunology, 250(1-2): 55-67.

[39] Mills, K. (2009): Designer adjuvants for enhancing the efficacy of infectious disease and cancer vaccines based on suppression of regulatory $\mathrm{T}$ cell induction. Immunology letters, 122: 108-111.

[40] Morelli, A., Larregina, A., Ganster, R., Zahorchak, A., Plowey, J., Takayama, T., Logar, A., Robbins, P., Falo, L., and Thomson, A. (2000): Recombinant Adenovirus induces maturation of dendritic cells via an NF-Kappa $b$ dependent Pathway. Journal of virology, 74(20): 9617- 9628.

[41] Pichla-Gollon, S., Lin, S., Hensley, S., Lasaro, M., Herkenhoff-Haut, L., Drinker, M., Tatsis, N., Gao, G., Wilson, J., Ertl, H. and Bergelson, J. (2009): Effect of preexisting immunity on an adenovirus vaccine vector: in vitro neutralization assays fail to predict inhibition by antiviral antibody in vivo. Journal of virology, 83: 5567-5573. 
[42] Ramos, O., Pose, A. Gomez-Puerta, S., Gomez, J., Redondo, A., Benites, J., Amaran, L., Parra, N. and Alonso, T. (2011): Avian CD154 Enhances Humoral and Cellular Immune Responses Induced by an Adenovirus VectorBased Vaccine in Chickens. Comparative immunology, microbiology and infectious diseases, 34(3): 259-265.

[43] Rea, D. (2001): Highly efficient transduction of human monocyte-derived dendritic cells with subgroup B fiber-modified adenovirus vectors enhances transgene-encoded antigen presentation to cytotoxic T cells. Journal of immunology, 166: 5236-5244.

[44] Rodriguez, L. and Grubman, M. (2009): Foot and mouth disease virus vaccines. Vaccine, 27(4):90 4.

[45] Russel, D. (2001): Update on adenovirus and its vectors. Biomolecular Sciences, School of Biology, University of St. Andrews, North Haugh, St. Andrews

[46] Saito, I., Oya, Y., Yamamoto, K., Yuasa, T. and Shimojo, H. (1985): Construction of non defective adenovirus type 5 bearing a 2.8kilobase hepatitis B virus DNA near the right end of its genome. Virology journal, 54: 711719.

[47] Samuel, K. and Michael, A. (2007): Current advances and future challenges in Adenoviral vector biology and targeting. Gene therapy, 7(3): 189

[48] Schnell, M. (2001): Activation of innate immunity in nonhuman primates following intraportal administration of Adenoviral vectors. Molecular therapy, 3:708-722.

[49] Shen, A. (2009): The U.S. Vaccine and Immunization Enterprise: Working to sustain and foster vaccine innovation. Human vaccines, 5(10): 649- 653 .

[50] Swayne, D. (2009): Avian influenza vaccines and therapies for poultry. Comparative immunology, microbiology and infectious disease, 32 (4): 351-363.

[51] Trotman, L., Mosberger, N., Fornerod, M., Stidwill, R. and Greber, U. (2001): Import of Adenovirus DNA involves the nuclear pore complex receptor CAN/Nup214 and Histone H1. Nature cell biology, 3(12): 1092- 1100.
[52] Uematsu, S. and Akira, S. (2007): Toll-Like Receptors and Type I Interferons. Journal of biological chemistry, 282(21): 15319-15323.

[53] Van Olphen, A. and Mittal, S. (1999): Generation of infectious genome of bovine adenovirus type 3 by homologous recombination in bacteria. Journal of virology methods, 77: 125-9.

[54] Verdino, P., Witherden, D., Havran,W. and Wilson, I. (2010): The Molecular Interaction of CAR and JAML Recruits the Central Cell Signal Transducer PI3K. Science, 329: 12101214.

[55] Wei, Z. and Michael J. (2003): Requirement of the Adenovirus IVa2 Protein for Virus Assembly. Virology, 77: 3586-87.

[56] Wesley, R., Tang, M. and Lager, K. (2004): Protection of weaned pigs by vaccination with human adenovirus 5 recombinant viruses expressing the hemagglutinin and the nucleoprotein of $\mathrm{H} 3 \mathrm{~N} 2$ swine influenza virus. Vaccine, 22: 3427-3434.

[57] Wyatt, L., Earl, P., Xiao, W., Americo, J., Cotter, C., Vogt, J. and Moss, B. (2009): Elucidating and Minimizing the Loss by Recombinant Vaccinia Virus of Human Immunodeficiency Virus Gene Expression Resulting from Spontaneous Mutations and Positive Selection. Journal of virology, 83(14): 7176-7184.

[58] Yue, L. (2000): Adenovirus E1B 55-Kilodalton Oncoprotein Inhibits p53 acetylation. Molecular and cellular biology, 20(15): 5540.

[59] Zeshan, B., Zhang, L., Bai, J., Wang, X., Xu, J and Jiang, P. (2010): Immunogenicity and Protective Efficacy of a Replication Defective Infectious Bronchitis Virus Vaccine Using an Adenovirus Vector and Administered in ovo. Journal of virology methods, 166(1-2): 54-59.

[60] Zhao, J. (2003): Improved protection of rhesus macaques against intrarectal simian immunodeficiency virus SIV (mac251) challenge by a replication competent Ad5hrSIVenv/rev and Ad5hr-SIVgag recombinant priming/gp120 boosting regimen. Virology journal, 77: 8354-8365.

Citation: Biniam Tadesse, Biruk Tesfaye, Ashenafi Kiros. Review on Adeno Virus; As a Vaccine Vehicle. ARC Journal of Animal and Veterinary Sciences. 2017; 3(2):28-37. doi: dx.doi.org/ 10.20431/2455-2518.0302003.

Copyright: (C) 2017 Authors. This is an open-access article distributed under the terms of the Creative Commons Attribution License, which permits unrestricted use, distribution, and reproduction in any medium, provided the original author and source are credited. 